\title{
A Method for Reliability Assessment of Aircraft Structures Subject to Accidental Damage
}

\author{
Cary K. Huang ${ }^{*}$, and Kuen Y. Lin ${ }^{\dagger}$ \\ University of Washington, Seattle, WA, 98195-2400
}

\begin{abstract}
A method has been proposed to assess the reliability of aircraft structures subject to accidental damage using in-service damage data. The development of this method is motivated by the increasing use of composite materials in aircraft structures, which are particularly sensitive to impact damage. In addition, damage to composite structures may not be visible or may be difficult to detect. Based on the equivalent Level of Safety (LOS) concept, this method consists of two essential elements: a probabilistic assessment of detected damages and the probability of non-destructive inspection methods to detect such damage. Accidental damage threats are first characterized by identifying damage type, size, cause, and frequency for different aircraft models and structural components. The damage threats are then modeled by constructing histograms and detected damage probability distributions. Probability of detection models for various non-destructive inspection techniques are then used to calculate the equivalent Level of Safety. This approach can be used to design a new aircraft structure that is equivalently or more reliable as current designs. Furthermore, maintenance service guidelines and optimum inspection intervals may be estimated once design limit load is established. Engineers can use this methodology in the future as a guide to establish design and inspection criteria while considering safety risk and maintenance cost at the same time. The current reliability method relies on the completeness of the reported data on detected damage. Fleet service data currently available may not contain accurate information on damage size and the associated inspection method. In addition, damage reporting procedures vary from one operator to another, and damage data available for composite structures are limited. Therefore, sample representation of the extracted damage sizes and the probability of reporting are issues that must be addressed in order to apply the method to practical aircraft design. In this paper, $100 \%$ reporting and $100 \%$ sample representation is assumed. The results of the current reliability analysis method may give insight on general damage threats and future methods for designing and inspecting composite aircraft structures.
\end{abstract}

\section{Nomenclature}

$A$

$=$ random variable for damage size

$=$ damage size

$=$ design critical damage size

$=$ binary random variable for damage detection state ( 1 indicates damage is detected)

$=$ number of flaws of the same damage type

$=$ number of damage locations

$=$ number of damage types at the $\mathrm{i}^{\text {th }}$. location

$\begin{array}{ll}p(a) & =\text { actual damage probability density function } \\ p_{0}(a) & =\text { detected damage probability density function }\end{array}$

$P_{D}(a) \quad=$ probability of detection for damage size $a$

$N D I=$ non-destructive inspection

LOS = level of safety

$P(X) \quad=$ probability of $X$

$P F \quad=$ probability of failure

\footnotetext{
* Graduate Research Assistant, Department of Aeronautics and Astronautics, Box 352400, AIAA Student Member.

$\dagger$ Professor, Department of Aeronautics and Astronautics, Box 352400, AIAA Associate Fellow.
} 


$\begin{array}{ll}P O D & =\text { probability of detection } \\ \Gamma(\alpha) & =\text { gamma function } \\ \alpha & =\text { probability distribution model parameter } \\ \beta & =\text { probability distribution model parameter }\end{array}$

\section{Introduction}

$\mathrm{N}$ recent years, aircraft reliability and risk assessment are becoming more accepted in the areas of structural integrity and fleet management. ${ }^{1}$. As the use of composite materials in aircraft structures are becoming more widespread, a greater need exists to determine the reliability of aircraft structures subject to accidental damage. Foreign object damage (FOD), ground vehicle collisions and lightning strikes are but a few examples of accidental damage an aircraft structure must face during its operational lifetime. By using a nondeterministic approach, the structural failure risks associated with accidental damage events can be assessed quantitatively, allowing aircraft manufacturers, operators, and flight certification authorities to better evaluate and predict the damage tolerance and safety of an aircraft structure.

Current aircraft design and certification philosophies are based on deterministic approaches which use safety and knockdown factors for various design conditions such as moisture, temperature, loading, and damage. Because of higher scatter in composite material properties and sensitivity of composite structures to impact damage, traditional methods have led to very conservative designs and service guidelines for composite structures. In essence, the current approach assumes that a "worst-case scenario" for each design condition occurs simultaneously. The result is a substantial weight and cost penalty which null the durability, lightweight, and performance advantages of composites. ${ }^{2}$. The reliability assessment method presented in this paper enables the utilization of service experience to take full advantage of the benefits of using composites. The potential of this method to increase structural safety and reduce maintenance cost is not however, limited to composite structures.

Past reliability and structural risk studies have focused on fatigue of aging aircraft, which is mainly an issue unique to metal structures. ${ }^{1}$. Composite structures are fatigue and corrosion resistant, but are much more sensitive to damage threats such as hail, bird strikes and ground vehicle collisions because of brittle behavior during failure. Furthermore, there may be no visible evidence of damage to composite structures, even though significant internal damage has been sustained. Many probabilistic methodologies incorporating micromechanics, laminate theory, manufacturing defects, operating environment, and impact damage have been proposed for composite structures, but few however, have addressed the important issues of inspection intervals and damage detection capability. ${ }^{3,4}$

This paper presents a probabilistic method to determine structural component reliabilities suitable for aircraft design, inspection, and regulatory compliance. The method is based on the equivalent Level of Safety (LOS) concept, which consists of two essential elements: a probabilistic assessment of detected damages and the probability of non-destructive inspection methods to detect such damage. The present method is a quantifiable, datadriven process that allows the comparison of relative safety between dissimilar aircraft structures having different structural configurations and material systems. ${ }^{5}$

\section{Reliability Formulation}

Modern damage tolerance philosophies require that damage accumulated during the service life of a component be detected and repaired before the strength of the component is degraded beyond some design threshold. The Level of Safety concept, proposed by Lin et $\mathrm{al}^{5}$, is conveniently defined according to these criteria. The structural reliability assessment method currently presented is based on the equivalent Level of Safety formulation. ${ }^{5}$.

\section{A. Level of Safety Definition}

The discrete Level of Safety (LOS) for a single inspection event is defined as the compliment of the probability that a single flaw size larger than the critical flaw size for residual strength of the structure exists, and that the flaw will not be detected. This definition assumes that at a single inspection opportunity, only a single flaw is present, and does not grow with time. ${ }^{5}$ Expressed in terms of the probability of failure (PF), represented by a joint probability function of the detection state and damage size, the LOS is defined by:

$$
L O S=1-P F=1-P\left(A \geq a_{c}, D=0\right)
$$




$$
P F=\int_{a_{c}}^{\infty} p(a)\left[1-P_{D}(a)\right] d a
$$

Since the actual damage density $p(a)$ is unknown, it is substituted with known probabilities using Baye's Law. The probability of failure becomes:

$$
P F=\frac{\int_{a_{c}}^{\infty} \frac{p_{0}(a)}{P_{D}(a)}\left[1-P_{D}(a)\right] d a}{\int_{0}^{\infty} \frac{p_{0}(a)}{P_{D}(a)} d a}
$$

All of the configuration-specific information and inspection techniques assumed are contained within the parameters of the probability distributions. Hence, the reliability equation is independent of the particular damage mechanism being modeled. As a result, the choice of appropriate probability models is important to accurately describe the nature of uncertainty for the specific problem of interest. Typical models for the probability of detection $P_{D_{D}}(a)$ are log-odds or lognormal cumulative distributions, and typical models for the detected damage size $p_{0 .}(a)$ are Gamma or Weibull distributions. ${ }^{2}$.

Multiple damage types often occur on a structure simultaneously, each with its own characteristic probability of detection (POD) distributions. Each damage type could also have different LOS values at different locations on the structure. Thus, the overall LOS for the entire structure is the product of all the discrete LOS values for each damage type at each location in the structure. Eq. 4 is the cumulative Level of Safety for all damage events that can be detected at a single inspection opportunity, assuming that the hazard due to an individual flaw is independent of the existence of other flaws. ${ }^{5}$

$$
L O S=\prod_{i=1}^{N_{L}} \prod_{j=1}^{N_{T_{i}}}\left(1-P F_{i j}\right)^{n_{i j}}
$$

The current reliability formulation contains a few important assumptions and limitations. First, it is assumed that a single characteristic dimension can describe the damage mechanism being modeled such as a crack length or dent diameter. Also, the reliability calculated for a structure using this method assumes a single inspection event at a fixed point in time, and is therefore valid only at the instant of calculation. ${ }^{2}$. Finally, the reliability method in its current form does not account for damage growth that may occur due to cyclic loading of the structure and cannot deal with interaction between multiple damages, as may be the real case where residual strength of a structure is highly dependent upon the size and location of the damages relative to each other.

\section{B. Mathematical Constraints}

There are important statistical and numerical restrictions associated with the current reliability formulation. This is a consequence of the close relationship between the detected damage size, the actual damage size, detection probability, and inherent assumptions in the probability models. Details about these restrictions can be found in Ref. 5. The following constraints must be imposed on the damage probability distribution and detection models in order for convergence of the reliability calculation:

- The detected damage density $p_{0 .}(a)$ must smoothly approach zero as damage size approaches zero.

- The detected damage density $p_{0 .}(a)$ must approach zero at a faster rate than the probability of detection.

- Depending on the computer tool used for numerical integration, the zero term in lower limit of the integration constant (Denominator of Eq. 3) may need to be replaced by a variable approaching zero in order to avoid an improper integral.

In most cases, these conditions are automatically satisfied. For special cases where the reliability calculation does not automatically converge, modification of the damage data is required. This topic will be discussed in more detail in the Reliability Assessment section. 


\section{Damage Characterization}

During operation and maintenance service, aircraft structures are subject to a variety of accidental damage threats. It is important to properly characterize these threats to assess a structure's reliability and damage tolerance capability. From many years of service experience, sources of accidental damage on aircraft structures have been identified and categorized, and are fairly consistent throughout the range of aircraft types and operating conditions of both military and civilian aircraft. ${ }^{2}$. Nonetheless, no generally-accepted method currently exists to measure and predict the structural damage extent or damage severity due to these known sources.

Many research efforts have tried to find a quantifiable correlation between impact energy and the residual strength of composite structures, since discrete-source impact damage is often the critical damage mechanism. Current damage tolerance and certification criteria for composite aircraft structures are based on these studies, using conservative probabilistic distributions of impact energies to describe damage threat and surface deformation to measure damage extent. There are however, considerable limitations to the use of impact energy and damage depth to characterize damage. Factors such as structural geometry, structural support conditions, and impactor shape also influence the damage severity, making it difficult to probabilistically assess damage without extensive testing and analysis of all possible configurations exposed to impact. In addition, probabilistic impact energy criteria are difficult to validate on operational composite structures since the only observable result of an impact event is the resulting damage, and not the impact energy. ${ }^{2}$

In the current reliability approach, damage is not characterized directly in terms of damage threat itself such as impact probability, but instead uses the size of the subsequent damage for probabilistic assessment. This allows a variety of damage threats such as lightning and maintenance errors, not just impact-related damage, to be considered for analysis. Consequently, under the current method, the damage threats and damage size probability distributions remain specific to the structural component or zone under assessment. To deal with this, damage maps can be constructed from available data to assist in zoning the aircraft into regions where the impact threat and damage resistance characteristics are consistent, or to identify areas that are particularly susceptible to large and/or frequent damage. Damage size distributions models can then be obtained for each zone or structural component identified from the damage maps, and combined to characterize damage on the overall aircraft structure. By using the current method, there is no need for a direct correlation of damage threat to damage severity, thus avoiding the consideration of many configuration-specific variables that add complexity to the reliability analysis.

\section{A. Damage Data}

Between the thousands of damage events contained in current databases and the many years of combined experience of aircraft maintenance personnel and engineers, a significant amount of resources are available to us for damage characterization. Much insight has already been revealed into the nature of accidental damage threats and the hazards they pose to the structural integrity of an aircraft structure. Yet, little quantitative information for reliability assessment purposes is available. There is much work to be done in the area of database development before non-deterministic methods can achieve their full potential. In this section, a few sources of currently available data are presented, and their limitations are described. Suggestions by the author to more efficiently organize damage data for characterization are also introduced. Ultimately, the goal is to achieve a working database from which aircraft designers, engineers, and operators can gain intelligent information about the safety and reliability of their aircrafts.

One source of publicly available data is the Federal Aviation Administration's (FAA) Service Difficulty Reporting System (SDRS) database. By requirement, commercial aircraft operators must submit their aircraft to periodic inspection and report any failure, malfunction, or defect that threatens flight safety or exceeds allowable limits. The SDRS database may be useful for damage characterization, but it is important for certain limitations to be taken into account before using SDRS data for statistical analysis. Since damage below maximum acceptable limits is not usually reported, the damage sizes extracted from the SDRS database may represent only large damage, not the overall damage size distribution of a component. In addition, not all incidences of major structural damage that occur on a component in service are filed into the database because repair of such damage is often handled through the FAA's Designated Engineering Representative or directly by the airframe manufacturer. These factors must be carefully considered in order to avoid significantly biased results. ${ }^{6}$

Ref. 4 is another source of damage data that may be used for characterization. Although the amount of data is limited, it can provide valuable information because it is one of the few published sources of composite damage data. This data was collected via survey from on-site inspections of several commercial airline maintenance facilities and a U.S. Naval Aviation depot. An alternative to using public data for damage characterization is the use of databases from private sources such as aircraft operators who keep logbooks of maintenance actions. Data from 
these sources may have more statistical value since all damage that requires repair (large or small, frequent or infrequent) are recorded. There is unfortunately no guarantee that these data will be obtainable since proprietary information restrictions may apply, and there is no one authority that all operators report to for logbook data collection.

As previously mentioned, quality damage data must first be acquired in order for accurate damage characterization. Damage information required for a thorough reliability assessment include damage type, damage size, cause, frequency, damage location and the method of detection. Unfortunately, currently available databases are not designed for this type of application. Existing data often does not contain damage sizes, or is not recorded using the same units of measurement. Also, the structural component subjected to damage is often not specified in detail. The lack of important damage information and the inconsistency in their descriptions present a major challenge for proper damage characterization, requiring significant resources to mine, categorize, and reorganize data. Besides being labor-intensive, this process also introduces additional uncertainty. For example, damage events that do not contain damage sizes have to either be excluded from the analysis, or a typical damage size must be assumed from the other available information. Still, damage threats and damage severity can be accurately characterized as long as the sample size of "good" data is sufficient.

In order to prepare available damage data for characterization and reliability assessment, careful procedures must be developed to assure that a database includes damage information that is complete and is categorized in such a way that is consistent. Unfortunately no industry standard exists for damage collection and reporting at present. Table 1 is an example of categories for damage data organization suggested by the author that would be ideal for current purposes.

Table 1. Example Categories for Damage Data Characterization

\begin{tabular}{|c|c|c|c|c|c|}
\hline $\begin{array}{l}\text { Material } \\
\text { Type }\end{array}$ & $\begin{array}{l}\text { Exterior } \\
\text { Damage }\end{array}$ & Damage Type & $\begin{array}{l}\text { Aircraft } \\
\text { Location }\end{array}$ & $\begin{array}{l}\text { Detection } \\
\text { Method }\end{array}$ & Damage Cause \\
\hline Metal & Yes & Crack & Fuselage & $\begin{array}{c}\text { Visual } \\
\text { Inspection }\end{array}$ & Birdstrike \\
\hline \multirow[t]{7}{*}{ Composite } & No & Dent & Wing & Tap Hammer & Ground Collision \\
\hline & & Delamination & $\begin{array}{l}\text { Horizontal } \\
\text { Stabilizer }\end{array}$ & $\begin{array}{l}\text { Mechanical } \\
\text { Impedance }\end{array}$ & $\begin{array}{l}\text { Departed Aircraft } \\
\text { Component }\end{array}$ \\
\hline & & Hole & $\begin{array}{l}\text { Vertical } \\
\text { Stabilizer }\end{array}$ & Ultrasonic & Runway Debris \\
\hline & & Other & Engine & Other & $\begin{array}{c}\text { Tire Tread } \\
\text { Separation/Blowout }\end{array}$ \\
\hline & & & $\begin{array}{l}\text { Landing } \\
\text { Gear }\end{array}$ & & Ice/Sleet/Hail \\
\hline & & & & & Lightning \\
\hline & & & & & Unknown \\
\hline
\end{tabular}

Note that a single damage event may contain multiple damage sizes, so each available damage size should contain category information corresponding to those suggested in Table 1. Furthermore, the categories for characterization are not limited to those listed, and can be further subcategorized.

\section{B. Damage Mapping}

With categorized damage data in hand, damage maps can be readily produced to identify structural zones with similar damage threats and damage resistance. A graphical representation of the spatial distribution and frequency of damage is a way to show the trends that are associated with each damage category. Thus through damage mapping, areas of a structure that are susceptible to a specific type of damage or are consistently damaged due to a known cause are immediately revealed. The areas with common damage characteristics can then be divided into zones for damage size distribution modeling. 
The damage mapping procedure is as follows: First, the aircraft structure of interest is identified and the structural geometry is assembled electronically in either two or three-dimensions. Next, the damage types, causes, and any other applicable damage categories of interest are selected for characterization. One or all of the variables within each category may be selected. Then, after the data of interest is extracted from the database, a damage size count is conducted and mapped onto the geometry. The advantage of using this procedure is that it is relatively easy to compare damage variables, assuming the data is well-categorized. Different damage causes, damage types, and inspection methods can all be independently or simultaneously compared. Another application for damage maps may be to identify geographic trends in damage by comparing accumulated damage on particular aircrafts servicing in different parts of the world.

Depending on the purpose of analysis, the level of detail of available data, and the amount of resources available to engage in this task, a damage map may be constructed with detail. To demonstrate, a rectangular wing was selected and mapped using assumed data $\$$. Fig. 1 illustrates a detailed map, displaying damage occurrence according to component stations. Note that the spots indicating damage do not reflect the actual size of the damage. Instead, the mapping was conducted using interpolation to show effects of neighboring damage. This method can be very useful in zoning the structure.

If the damage data is not recorded in detail, a damage map may be constructed for discrete structural zones. Fig. 2 illustrates damage occurrence on the same rectangular wing for typical regions such as the leading edge, flap, aileron, wing tip, and main wing body. Corresponding histograms and probability distributions for each region can then be constructed after damage sizes have been extracted.

Damage mapping enables engineers to identify structural components that are at high risk to encounter major structural damage or require frequent repair. Airframe designers can take advantage of this information and tailor their designs accordingly to avoid over-designs and costly maintenance repair. The overall result is a more efficient structure that has extra damage tolerance capability in areas of high damage risk and significant weight savings in areas of low damage risk.

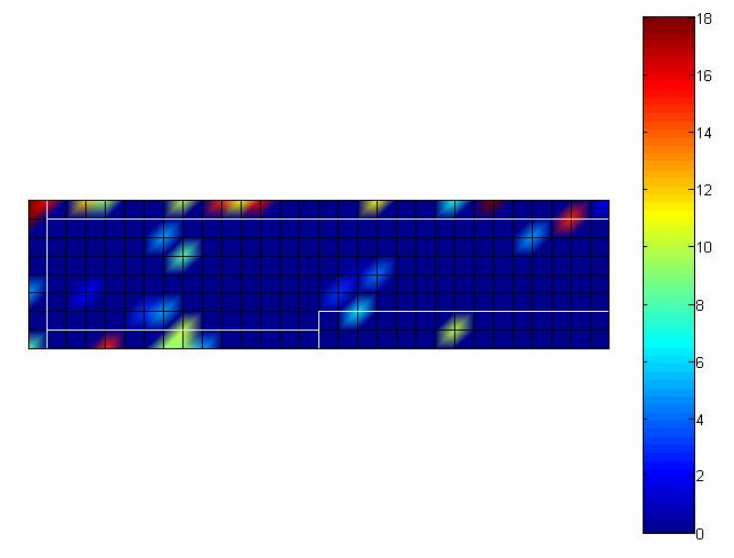

Figure 1. Detailed Damage Map of Rectangular Wing

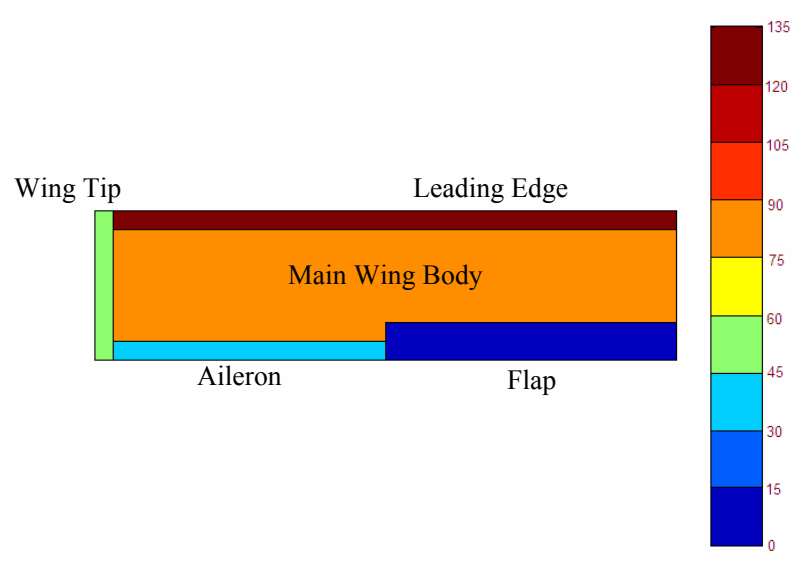

Figure 2. Discrete Damage Map of Rectangular Wing

\section{Detected Damage Probability Distributions}

Once the damage data is properly categorized and aircraft structure appropriately zoned, detected damage size probability distributions can be constructed. A number of numerical analysis tools have the ability to calculate maximum likelihood estimators (MLE's) for the probability distribution parameters, once a probability model is assumed. The selection of appropriate probability models for detected damage characterization is very important because the reliability is calculated directly from the probability distribution functions (PDF's), and the choice of one over another may dramatically change the reliability assessment results. Experience has shown that twoparameter Gamma and Weibull distributions model damage sizes found during maintenance inspections quite

$\$$ Data used for demonstration purposes are generated from assumed probability distributions typical to those encountered in real service. 
accurately. Both probability models are very flexible to assume a wide range of shapes according to the damage data. The PDF for the Gamma and Weibull distributions are shown in Eqs. 5 and 7. Other probability models may be used as well, and parameters may be obtained in the same manner. In order to determine which probability model best fits the damage size data under characterization, a goodness-of-fit test must be performed for each of the probability models of interest. ${ }^{2}$.

1. Gamma Distribution

$$
\begin{gathered}
p_{0}(a)=\frac{1}{\Gamma(\alpha) \beta^{\alpha}} a^{\alpha-1} e^{-a / \beta} \\
\Gamma(\alpha)=\int_{0}^{\infty} t^{\alpha-1} e^{-t} d t
\end{gathered}
$$

\section{Weibull Distribution}

$$
p_{0}(a)=\frac{\alpha}{\beta} a^{\alpha-1} e^{-a^{\alpha} / \beta}
$$

Characterization of the detected damages requires two simple steps: First, the structural component and damage types to be assessed are selected, and the corresponding damage size data extracted. Then after a probability model is assumed and the damage size range to be considered is defined, the distribution parameters are obtained using numerical data fitting tools. The rectangular wing example from Fig. 2 will be used to demonstrate. Let us assume the aileron is constructed of a composite sandwich structure similar to those currently used in commercial aircraft, and the goal is to assess the reliability of this component subjected to crack and delamination damage. Using the same assumed data from before, damage sizes between zero and ten inches were extracted for the two damage types specified, and fitted to a Gamma distribution. The PDF parameters are shown in Table 2 and plotted in Fig. 3.

In order for accurate characterization of

Table 2. Gamma Distribution Parameters for Rectangular Wing Aileron

\begin{tabular}{l|l|l}
\hline \hline Damage Type & $\boldsymbol{\alpha}$ & $\boldsymbol{\beta}$ \\
\hline Crack & 1.95 & 2.09 \\
\hline Delamination & 4.23 & 1.40 \\
\hline \hline
\end{tabular}

the detected damages, the sample size of damage data must be sufficient. "Kinks" or irregularities in the fitted PDF are easy indications of insufficient data. It is difficult to estimate exactly how many damage sizes are necessary, but from experience, a sample size of one hundred or more is typically the minimum. In cases where not enough data exists, Bayesian statistical tools can be used to update the damage size probability distributions when new data become available. Research by Rusk et al on this subject can be found in Ref. 6 .

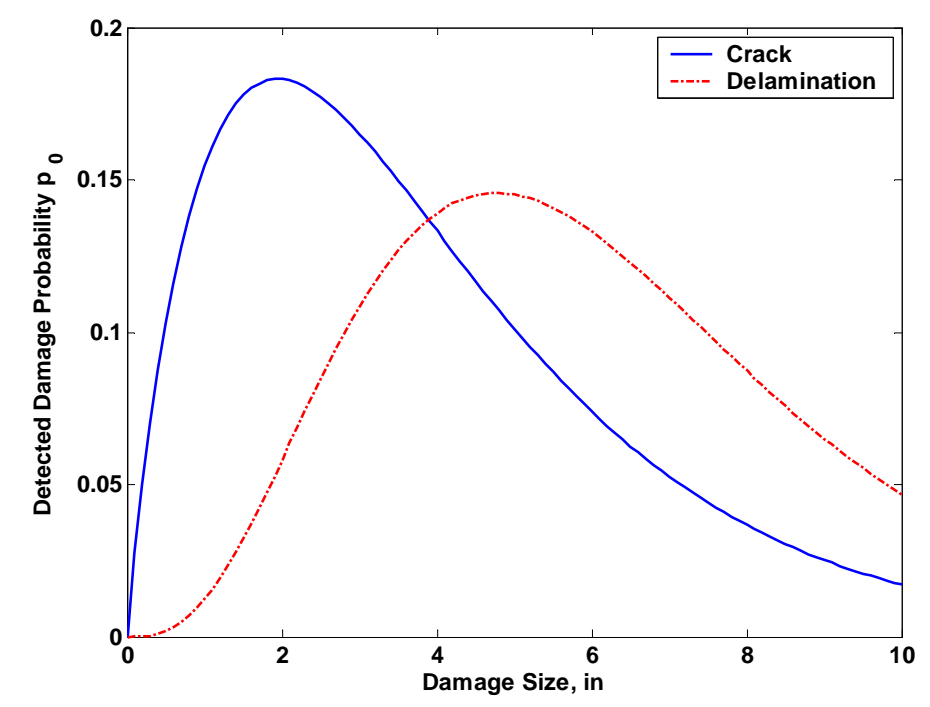

Figure 3. Detected Damage Size Gamma Distribution of Rectangular Wing Aileron

\section{Probability of Detection}

Significant research has been conducted to quantify the capability of various non-destructive inspection (NDI) methods to detect damage. Although the majority of these studies have focused on constructing Probability of 
Detection (POD) models for cracks in metal structures, recent experiments on composite structures show similar results. Research shows that a cumulative LogNormal distribution or Log-Odds approximation can be used to model the mean "hit/miss" response from these experiments. ${ }^{7}$. Because the LogNormal distribution does not have a closedform solution, the Log-Odds POD model described by Eq. 8 proves to be more convenient.

$$
P O D(a)=\frac{e^{\alpha+\beta \ln (a)}}{1+e^{\alpha+\beta \ln (a)}}
$$

Two recent studies have been conducted to characterize POD models for composite structures. One is a joint effort between the FAA's Airworthiness Assurance Center, Sandia National Laboratories, and the Commercial Aircraft Composite Repair Committee (CACRC) to quantify the performance of current NDI techniques in detecting subsurface composite damage, and the degree of improvements possible through the integration of more advanced techniques and procedures. Some of the NDI tools used for the experiment are the tap hammer, mechanical impedance, shearography, and thermography testing machines. ${ }^{7}$ The other is an experiment conducted by a private aerospace company to determine the visual inspection capability to detect surface dents from impact. Both studies focus on important aspects of damage detection because visible surface damage and invisible subsurface damage alike, pose a threat to the structural integrity of an aircraft. Also, both studies consider variables such as inspector variation and environmental conditions during inspection.

Caution needs to be used before incorporating the POD's from these experiments into reliability assessment because all experiments were carried out under carefully monitored conditions on flat composite panels. Inspectors performing under the experiment are looking for damage that they know exist, which is very different from a walkaround visual inspection or a structural maintenance inspection on a much larger and more complex aircraft structure. In addition, many other variables such as lighting conditions, structural curvature, inspector training, and available inspection tools are present when inspecting a real aircraft structure for damage. Therefore, the damage detection probability is expected to be much higher for smaller damage sizes due to

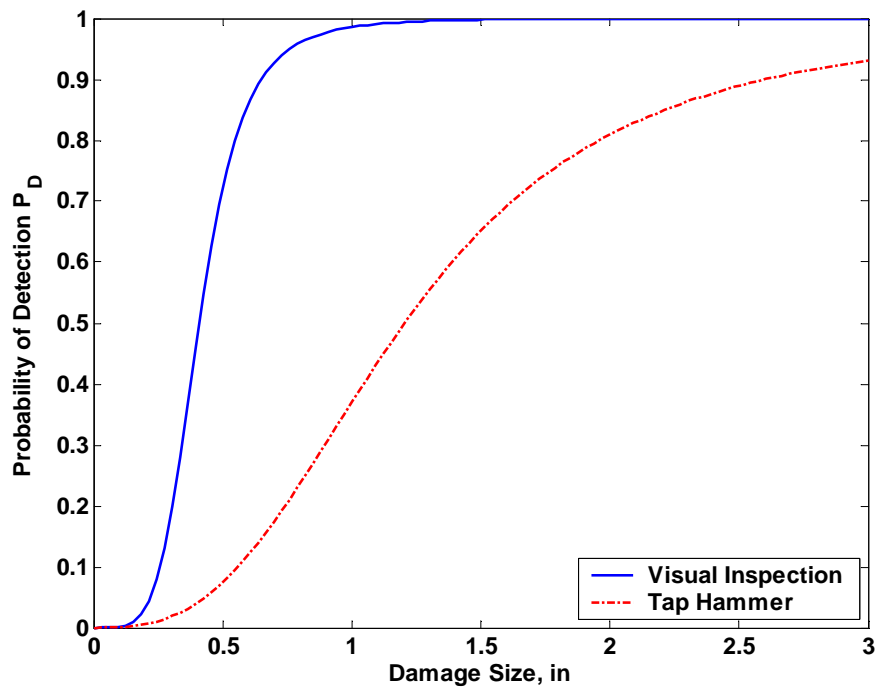

Figure 4. POD for Visual and Tap Hammer Inspection Methods special experimental conditions.

For the purpose of demonstration, a conservative approach will be used since information on the method of inspection used to detect damage is rarely available. Also, not all maintenance facilities are equipped with advanced NDI tools for damage detection. Therefore, visual and tap hammer inspections methods are assumed. Table 3 contains typical parameters to Eq. 8 for the two selected inspection methods, and POD distributions are shown in Fig. 4.

Table 3. POD Parameters for Visual and Tap Hammer Inspection Methods

\begin{tabular}{l|l|l}
\hline \hline Inspection Method & $\alpha$ & $\beta$ \\
\hline Visual & 4.22 & 4.69 \\
\hline Tap Hammer & -0.55 & 2.86 \\
\hline \hline
\end{tabular}




\section{Reliability Assessment}

With detected damage distributions and POD's ready, the final step is calculating the reliability of the structure of interest. Following the example from previous, the structural component under assessment is an aileron of a rectangular wing subject to crack and delamination damage, assuming visual and tap hammer as methods of inspection. The Level of Safety at a single inspection opportunity for each combination of damage type and inspection method is calculated using Eqs. 1 and 3 , and combined using Eq. 4 to determine the cumulative LOS of the structural component. The result is shown in Fig. 5.

Figure 5 shows that for a design critical damage size of four inches, there is less than $1 \%$ probability that crack and delamination damage to the structure will occur and will not be detected by inspection via the visual and tap hammer inspection methods. The LOS can be cross-plotted with results from residual strength analysis to produce design charts of the Level of Safety as a function limit load and the structural sizing parameter of laminate thickness. ${ }^{5}$. The same analysis can also be used to determine inspection intervals, using the same damage data and inspection capabilities to derive the likelihood that an accidental damage size will exceed a critical design size corresponding to the limit load over time.

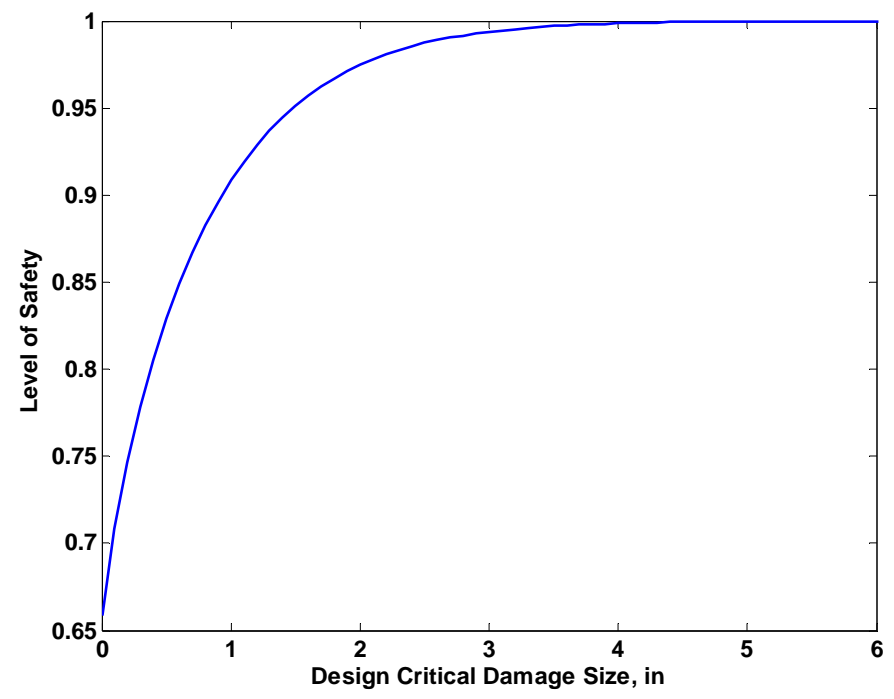

Figure 5. Cumulative Level of Safety for Aileron Subject to Crack and Delamination Damage Assuming Visual and Tap Hammer Detection Methods

In some cases where the mathematical

constraints defined previously are not automatically satisfied, special procedures must be followed in order for convergence of the reliability calculation. One way is to remove damage sizes less than a certain detection limit such as damage sizes less than 0.5 inches, and obtain new detected damage distribution parameters. In doing so, we automatically assume that damage sizes less than 0.5 inches are simply not detected or not large enough to be a safety matter, so therefore not recorded. This action leads to conservatism in the analysis, but should not influence result too much as long as damage detection probability is not high for the damage size range to be removed. In practice, the selection of this damage size may correspond to design criteria such as the barely visible impact damage (BVID) limit or allowable damage limit (ADL). Nonetheless, caution must be taken when making such assumptions because detected damage size and detection capability are directly related.

\section{Conclusion}

By taking a probabilistic approach to structural failure, the need for arbitrary factors of safety based on traditional design practices is substantially reduced. The reliability assessment method presented enables aircraft manufacturers, operators, and certification authorities to quantitatively evaluate the structural failure risks associated with accidental damage events while considering costs as well. By using this method during structural design and maintenance planning, the full benefits of using composite structures may be realized.

In this paper, the procedure for reliability assessment was demonstrated using data typical to those encountered in real service. Considerations for proper structural zoning, damage characterization, and detection capability determination were also described. The current reliability method relies on the completeness of the reported data on detected damage. Unfortunately, available in-service data may not contain complete descriptions of damage size, structural locations and inspection method. In addition, operator damage reporting procedures often vary. In this paper, $100 \%$ reporting and $100 \%$ sample representation is assumed. The sample representation of the extracted damage sizes and the probability of reporting are issues that must be addressed in order to apply the method to practical aircraft design. 


\section{Acknowledgments}

The authors wish to thank The Boeing Company and the FAA Center of Excellence at the University of Washington (AMTAS) for sponsoring the current research project. Thanks also to Dr. Cliff Chen, Dr. Hamid Razi, and Dr. Andrey Styuart for the invaluable discussions in the development of the reliability method presented.

\section{References}

${ }^{1}$ Tong, Y. C., "Literature Review on Structural Risk and Reliability Analysis," Aeronautical and Maritime Research Laboratory, Rept. DSTO-TR-1110, Melbourne, Australia, Feb. 2001.

${ }^{2}$ Rusk, D. T., "Reliability-Based Methods Applied to the Design of Damage Tolerant Aircraft Structures," M.S. Thesis, Dept. of Aeronautics and Astronautics, Univ. of Washington, Seattle, WA, June 2000.

${ }^{3}$ Long, M. W., and Narciso, J. D., "Probabilistic Design Methodology for Composite Aircraft Structures," U.S. Dept. of Transportation/Federal Aviation Administration, Rept. DOT/FAA/AR-99/2, Washington, DC, June 1999.

${ }^{4}$ Gray, P. M., and Riskalla, M. G., "Development of Probabilistic Design Methodology for Composite Structures," U.S. Dept. of Transportation/Federal Aviation Administration, Rept. DOT/FAA/AR-95/17, Washington, DC, Aug. 1997.

${ }^{5}$ Lin, K. Y., Du, J. and Rusk, D. T., "Structural Design Methodology Based on Concepts of Uncertainty," National Aeronautics and Space Administration, Rept. CR-2000-209847, Langley, VA, Feb. 2000.

${ }^{6}$ Rusk, D. T., Lin, K. Y., Swartz, D. D., and Ridgeway, G. K., "Bayesian Updating of Damage Size Probabilities for Aircraft Structural Life-cycle Management,” AIAA Journal of Aircraft, Vol. 39, No. 4, July 2002, pp. 689-696.

${ }^{8}$ Roach, D., and Rackow, K., "Improving In-Service Inspection of Composite Structures - It's a Game of CATT and MAUS," U.S. Dept. of Defense/National Aeronautics and Space Administration/Federal Aviation Administration Aging Aircraft Conference, Sept. 2003. 